\title{
Influences on the excitation behavior of lightweight planetary gearboxes
}

\author{
Julian Theling ${ }^{1,2}$. Jens Brimmers ${ }^{1}$ Christian Brecher ${ }^{1}$ \\ Received: 1 April 2021 / Accepted: 24 June 2021 / Published online: 13 September 2021 \\ (c) The Author(s) 2021
}

\begin{abstract}
One of the main cost drivers in mechanical engineering is material. In order to reduce costs, but also to reduce $\mathrm{CO} 2$ emissions in mobile applications, increasing efforts are being made to reduce the mass of components. In gear development, power density as the ratio of transmittable power to gear weight has become one of the key quality criteria. However, saving material usually also leads to lower stiffness of the components. In combination with higher specific powers and the associated higher forces, higher deformations and displacements occur in the gear unit. The gear unit is more elastic overall.

Within this paper a measuring cell is presented which allows to measure the influence of elastic deformations and additional misalignments on the excitation behavior. By means of this measuring cell, the influence of certain misalignments and different ring gear rim thicknesses are investigated. The results show modulated excitations and sidebands which are excited depending on the misalignment.
\end{abstract}

\section{Einfluss von Leichtbau auf das Anregungsverhalten von Planetengetrieben}

\section{Zusammenfassung}

Einer der wesentlichen Kostentreiber im Maschinenbau ist das Material. Zur Kostenreduktion aber auch zur Reduktion des $\mathrm{CO} 2$ Ausstoßes bei mobilen Anwendungen wird zunehmend versucht die Masse der Komponenten zu verringern. In der Getriebeentwicklung hat sich die Leistungsdichte als Verhältnis von Getriebegewicht zur übertragbaren Leistung als eines der wesentlichen Qualitätskriterien manifestiert. Das Einsparen von Material führt in der Regel aber auch zu geringeren Steifigkeiten der Komponenten. In Kombination mit höheren spezifischen Leistungen und damit einhergehenden höheren Kräften treten höhere Verformungen und Verlagerungen im Getriebe auf. Das Getriebe ist insgesamt elastischer. In diesem Bericht wird eine Messzelle vorgestellt, die es ermöglicht die Einflüsse der elastischen Verformung sowie von zusätzlichen Achslageabweichungen in Planetengetrieben auf das Anregungsverhalten zu untersuchen. Mit Hilfe der Messzelle wird der Einfluss unterschiedlicher Hohlradkranzdicken und Verlagerungen untersucht. Die Ergebnisse zeigen in Abhängigkeit der aufgeprägten Achslageabweichung unterschiedlich modulierte Anregungen und Seitenänder.

\section{Introduction and motivation}

Due to the distribution of power over several paths, planetary gear stages have a higher power density than a cylindrical gear stage and is thus increasingly used despite its

Julian Theling

j.theling@wzl.rwth-aachen.de

1 Laboratory of Machine Tools and Production Enginnering (WZL), RWTH Aachen University, Campus Boulevard 30, 52074 Aachen, Germany

2 RWTH Aachen University, Aachen, Germany increased design complexity. To further increase the power density, material can be reduced in particular in the lowstressed components such as the planet carrier. However, the lower stiffness then leads to higher elastic deformations, see Fig. 1.

In the planet carrier, the primary deformation is torsion. In addition, there are deformations of the carrier walls caused by the tilting moment of the planet gear. Deformations of the planetary gear shaft result from the gear forces. This results in axial position deviations of the planetary gear as well as pin hole errors and center distance changes. 
Fig. 1 Influence of high power densities on the elastic deformation

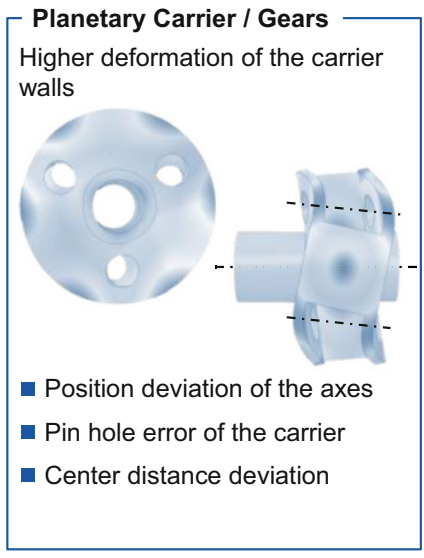

- Overall System

Higher deformation and deviation of the overall system

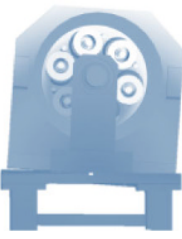

- Misalignment of the central elements sun gear, ring gear and planet carrier
Due to the large diameter, a comparatively large amount of material can be saved by reducing the ring gear thickness. The result is a lower mesh stiffness and greater deformation of the gear mesh. At the same time, the more flexible mesh also favors a better load distribution and load sharing of the gear meshes. In planetary gears with high power densities, there is also a trend toward a higher number of planets, so that the individual meshes are closer together locally. Deformations in the area of one mesh can thus affect the neighboring meshes, see Fig. 1 center.

Further elastic deformations can result from the gearbox environment. The environment has a particular effect on the axial position of the central shafts sun, ring gear, and planet carrier. Overall, therefore, gear boxes with high power densities are subject to higher elastic deformations, which affect the operating behavior and must therefore be taken into account during design.

\section{Objective and approach}

Planetary gearboxes react more sensitively to misalignments of the gears than simple cylindrical gear stages due to the additional influence on the load distribution. Due to the multiple meshes, not only the inclination and skew of the contact partners is decisive, but also the global position of the elements in relation to each other.

Investigations of the excitation behavior of planetary gearboxes show an influence of the phase position and long wave gear deviations on the excited frequencies. In the works of Inalpolat, Plöger and Morikawa, an analysis of the structure-borne noise signal at a static location on a stationary ring gear is carried out and modulations in the measured frequencies are found, especially in the region of the first gear mesh order [1-3]. The works of Inalpolat and Plöger partly contradict each other in the characteristics of the measured signal [1,3]. Frequency and amplitude modulation of the excitation signal are considered to be the cause of the modulation. Modulation effects in the transmission error could so far only be determined computationally in own reports and are otherwise not part of the state of the art [4].

Due to the increasing power density, stronger displacements and higher deformations of the gear mesh are to be expected, which thereby lead to excitations in the system. The effects of individual misalignments have not yet been

Fig. 2 Objective and Approach

$\begin{aligned} & \text { Challenge } \\ & \text { The effects of elastic deformations in planetary gearstages on the excitation behavior are not sufficiently well- } \\ & \text { known }\end{aligned}$
$\begin{aligned} & \text { Ocurring Effects } \\ & \text { Objective } \\ & \text { Development of a measuring } \\ & \text { behavior }\end{aligned}$


extensively investigated. Runout deviations of individual gears in planetary gear stages also lead to modulation effects, which excite further frequencies in the area of the gear mesh orders. Investigations whether these modulation effects can also be caused by misalignments of gears or elastic deformations are not available. Furthermore, all investigations were carried out with statically positioned structureborne noise sensors and the influence on the transmission error was not determined. Therefore, the objective of this report is to experimentally determine the effects of elastic deformations on the excitation behavior of planetary gears, Fig. 2.

Three steps are necessary to achieve the overall objective. In order to determine the effects of elastic deformation on the excitation behavior, a measuring cell must first be developed with which specific influencing variables can be specified. The influences mapped here are to include both specific applied displacements, as can occur in particularly elastic systems, and changes in the mesh stiffness. In a second step, the experiments will be carried out and the excitation behavior of the measuring cell in the reference state without additional displacements will be investigated. Here, the focus will be on the effects of lower mesh stiffnesses in particular. In a third step, the influence of additional displacements in the measuring cell is investigated. The in-

Fig. 3 Depicting elastic deformations at the test rig

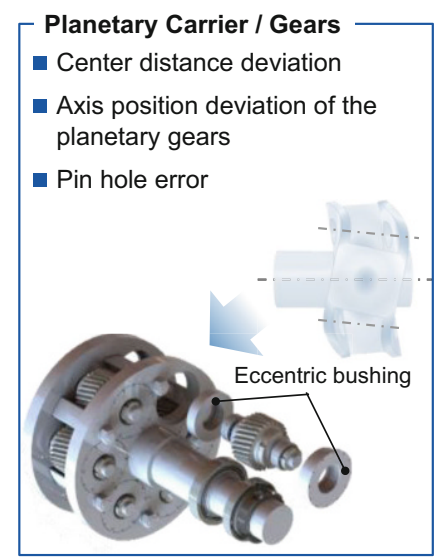

fluences of the misalignments are identified by comparison with the reference. An analysis is also carried out for different mesh stiffnesses.

\section{Presentation of the measurement cell for the investigation of influences from elastic deformations}

To investigate the influence of high elastic deformations on the excitation behavior in the tooth contact, a measuring cell is required which allows the effects of the reduced stiffnesses to be set in a specific way, see Fig. 1. On the one hand, displacements of the planetary gear are to be investigated as essential influencing variables from the environment, as they can be caused, by a deformation of the planet carrier, the planet pins or the bearing of the planet, cf. Fig. 3. The deviations resulting for the planetary gear are axial position deviations in form of inclination and skewing due to torsion and bending. Also, center distance deviations as well as pin hole errors can occur due to a deformation of the carrier walls or the planet shafts.

These effects are mapped on the test rig by means of a specific displacement of the planet gear shafts. For this purpose, the planets are designed as pinion shafts and sup-

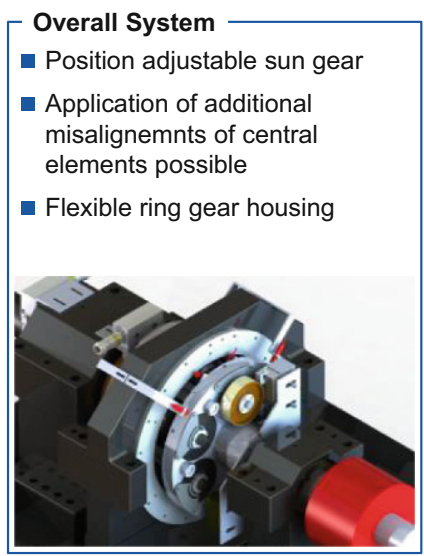

Fig. 4 Measuring cell and test setup

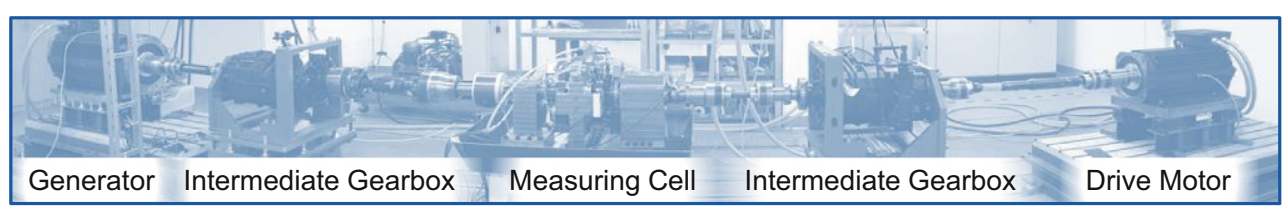

$2 x$

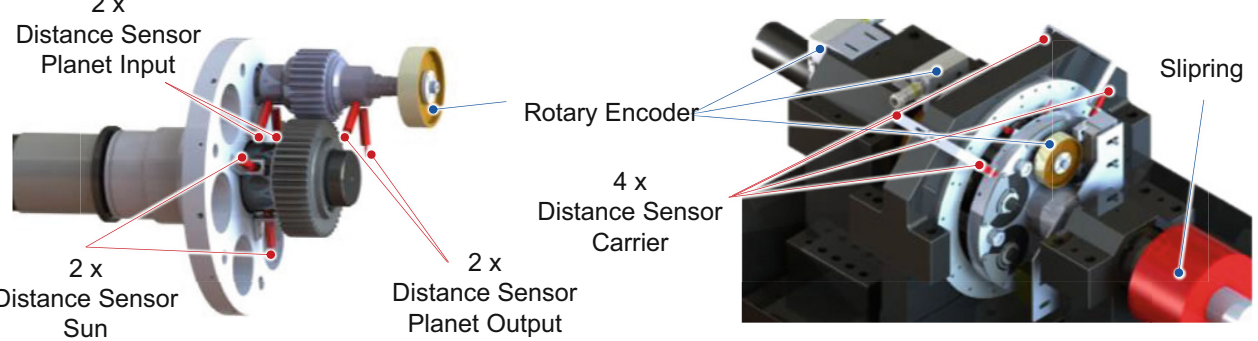


ported by eccentric bushings in combination with spherical roller bearings. By rotating the eccentric bushings relative to each other, additional axis position deviations can be set in a controlled manner. On the other hand, the influence of the mesh stiffness of the ring gear in particular must be analyzed, since in industrial practice the ring gear is often thinwalled and thus flexible due to load compensation effects and the possibility of a high weight reduction. In order to investigate different ring gears, the ring gear is connected to the housing via two flanges to the left and right of the geared area. This enables the mounting of ring gears with variable rim thicknesses without preventing deformation in the geared area due to the connecting structure.

Particularly in the case of elastic planetary gears, an influence of the number of planets in the system can be assumed. With a high number of planets, the meshes are spatially closer together and it is to be expected that the meshes interact with each other via a deformation of the central gears. In order to investigate variable numbers of planet gears, the carrier is constructed in a way that planets can be disassembled when the gearbox is assembled. To do this, the two eccentric bushings are loosened and the planet can be pulled out through the opening on the shaft side of the planet carrier, see Fig. 3 left.

In accordance with its widespread use in industrial practice, the measuring cell has an adjustable sun gear shaft. In addition, not only the ring gear but also its housing is designed to be comparatively flexible. The connection of the housing to the base plate via a base results in a low rigidity of the housing in the upper area. By moving the individual bearing blocks on the base plate, misalignments of the central elements can also be investigated, cf. Fig. 3 right.

The setup of the measuring cell is shown in Fig. 4 above. Due to the high torques to be investigated for the purpose of generating high deformations, intermediate gearboxes are used at the input and output. Constant velocity shafts are used to decouple the intermediate gearboxes from the measurement cell. The sun gear is driven by the input motor.

Fig. 5 Gear modifications of the helical gear set
Table 1 Gear data

\begin{tabular}{lll}
\hline & Spur & Helical \\
\hline Number of teeth sun $[-]$ & 47 & 43 \\
Number of teeth planet $[-]$ & 28 & 29 \\
Number of teeth ring $[-]$ & -103 & -101 \\
Center distance a [mm] & 112.5 & 112.5 \\
Module $\mathrm{m}_{\mathrm{n}}[\mathrm{mm}]$ & 3 & 3 \\
Transv. pressure angle $\alpha_{\mathrm{n}}\left[^{\circ}\right]$ & 20 & 20 \\
Helix angle $\beta\left[^{\circ}\right]$ & 0 & 12 \\
Gear width $[\mathrm{mm}]$ & 45 & 45 \\
\hline
\end{tabular}

Due to the higher torque of the generator the output is realised via the planet carrier. The maximum output torque is limited to $5.5 \mathrm{kNm}$ by the power of the generator.

The excitation behavior is measured via the transmission error. For this purpose, magnetic rotary encoders of the Baumer company of the type MHGE have been mounted on the sun gear, planet carrier and co-rotating on the planet gear. Due to the low pulse count of the magnetic encoders (64 or 128 pulses per rotation), post-processing of the measured sine-cosine signal via a Baumer HAEG interpolator is necessary to increase the number of pulses to 8192 per rotation. The pickup sensor of the encoder on the planetary gear is attached to the planet carrier and the signal is passed via a slip ring from the company Rie-Tech from the rotating planet carrier to the static DAQ system from the company Rotec. The signals are sampled with $50 \mathrm{kHz}$.

In addition to the excitation behavior, the displacements of individual gear elements are detected via inductive distance sensors from the company Contrinex of type DWAS. Two sensors measure the displacement of the sun gear in relation to the carrier. They are mounted on the planet carrier $90^{\circ}$ apart from each other and face the sun shaft. Four sensors measure the deformation and displacement of the planetary gear shaft in radial and tangential directions and are mounted on both carrier walls. In addition, the displacement of the planet carrier in comparison to the ring gear housing is determined via four further sensors. The

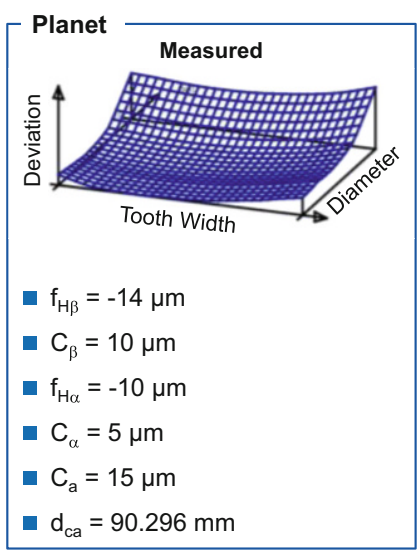

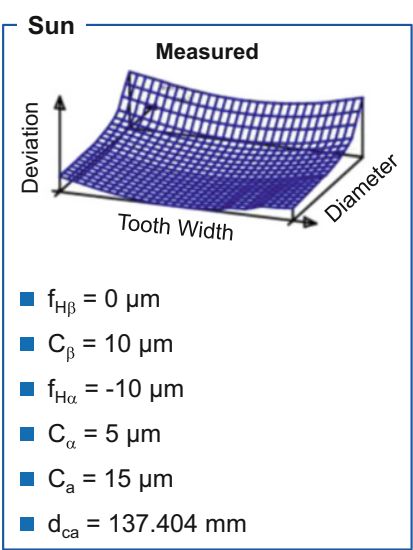

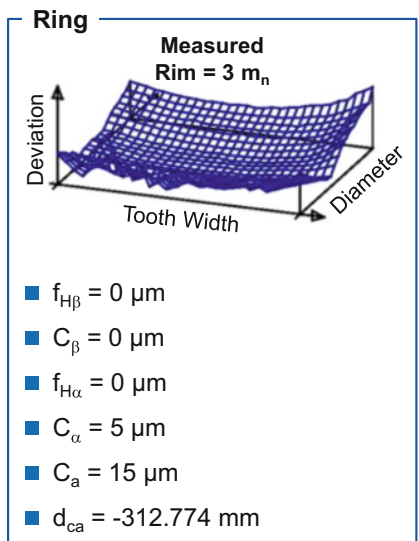


Fig. 6 Test plan and conditions
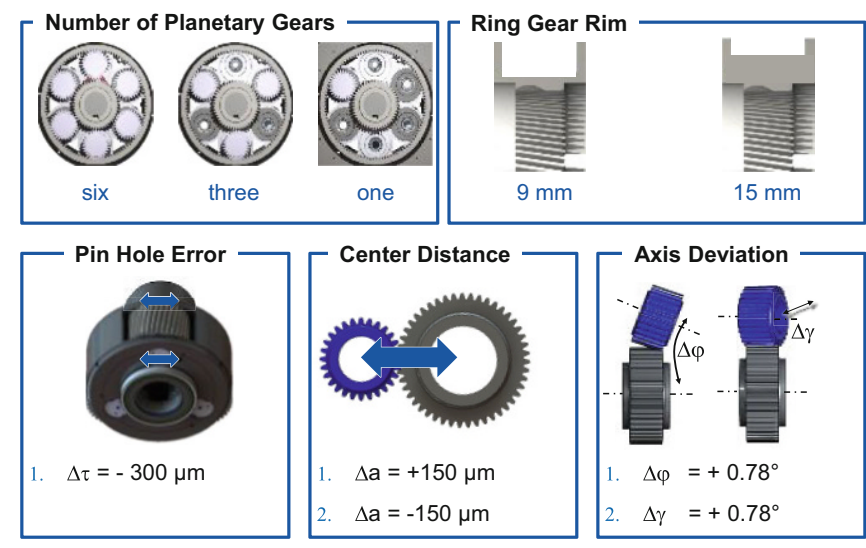

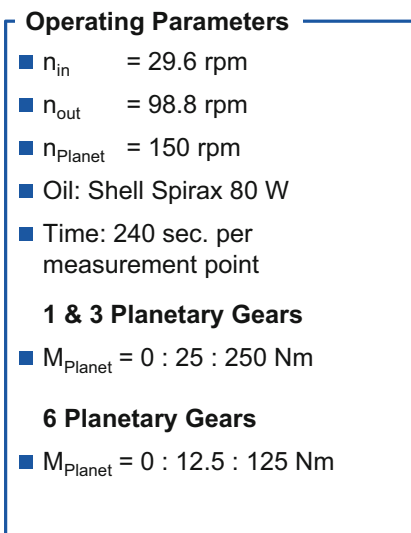

Operating Parameters

$\mathrm{n}_{\text {in }} \quad=29.6 \mathrm{rpm}$

- $\mathrm{n}_{\text {out }} \quad=98.8 \mathrm{rpm}$

- $\mathrm{n}_{\text {Planet }}=150 \mathrm{rpm}$

Oil: Shell Spirax $80 \mathrm{~W}$

Time: 240 sec. pe

M $M_{\text {Planet }}=0: 25: 250 \mathrm{Nm}$
6 Planetary Gears
$M_{\text {Planet }}=0: 12.5: 125 \mathrm{Nm}$

Sensors are mounted on the ring gear housing and face both carrier walls. The signals of the distance sensors are sampled with $1 \mathrm{kHz}$.

\section{Investigation of the influence of elastic deformations on the excitation behavior}

With the aid of the measuring cell presented in the previous chapter, a large number of tests are carried out. The test plan is shown in Fig. 6. In order to identify the influence of the multiple meshes on the individual components, the number of planetary gears is varied between one planetary gear, three planetary gears and six planetary gears. All investigations are also carried out for two different ring gear thicknesses. A spur gear and a helical gear are investigated. The gear data for the two variants can be found in Table 1 .

All gears are modified in order to reduce the sensitivity against misalignments and prevent premature tooth contact. The applied modifications of the helical gear stage are depicted in Fig. 5. Planet modifications are applied on both flank sides identically. All external gears were manufactured in house and tolerance classes 2 or better according to DIN ISO 1328-1 were achieved [5]. The two helical ring gears are modified identically. The manufacturing process was done externally and tolerance class 7 according to DIN ISO 1328-1 was achieved [5]. The spur gears are modified similar and show the same tolerance classes.

The ring gear thicknesses are selected so that they correspond to three times as well as five times the normal module, cf. Fig. 6. Only the thick-walled variant was manufactured for the spur gear. The eccentric bushes used have an eccentricity of $E=150 \mu \mathrm{m}$ with a deviation of $\Delta E=9 \mu \mathrm{m}$ measured. Initially, all eccentric bushings are aligned identically. The point of maximum eccentricity points in the tangential direction of the planet carrier, so that the center distance and the pin hole position correspond to those of an undisplaced planetary gear.
For the investigations of the influence of the misalignment, one planet is always deflected, the other planets remain in their initial position. By rotating the two eccentric bushings of one planet by $180^{\circ}$, a pin hole error of $\Delta \tau=300 \mu \mathrm{m}$ is set. This leads to an uneven distribution of the planets over the circumference of the carrier. To change the center distance, the two eccentric bushings of one planet are rotated in the same direction by $\pm 90^{\circ}$. Since the other planets remain undeflected, there is an additional pin hole error of $\Delta \tau=150 \mu \mathrm{m}$. Axis position deviations in pure tilt and skew direction are adjusted by the opposing rotation of the eccentric bushings. The achieved misalignment corresponds to $\Delta \varphi=\Delta \gamma=0.78^{\circ}$ in both planes.

The tests are carried out at a constant speed of $\mathrm{n}_{\text {Planet }}=150 \mathrm{rpm}$ and the torque related to the planet gear is increased stepwise in steps of $\Delta \mathrm{M}_{\text {Planet }}=25 \mathrm{Nm}$ from $M_{\text {Planet }}=0 \mathrm{Nm}$ to $250 \mathrm{Nm}$ for one and three planets. With six planets, the torque is halved in relation to the planet gear so that the same drive torque is applied at three and six planets.

\subsection{Modulation effects in the transmission error signal}

Due to the sequential mesh sequence of the two tested geometries, an excitation of the higher harmonics of the gear mesh order and not of the first gear mesh order (1.Og) is expected [6]. However, this cannot be confirmed in the test rig results. Here, for all variants, the highest excitation is in the range of the 1.Og. Additionally, modulations in the overall transmission error of the planetary gearbox were measured. These effects occur both in the displaced tests and in the reference tests. It should be mentioned here that deformations and deviations of the system are also present in the reference tests, but they are not additionally imposed. In a first step, the occurring modulation effects are described and the cause explained in order to be able to interpret the measurement results correctly. 
Fig. 7 Order spectrum of the helical gear stage in the range of the $1 . \mathrm{Og}$ without additional misalignments
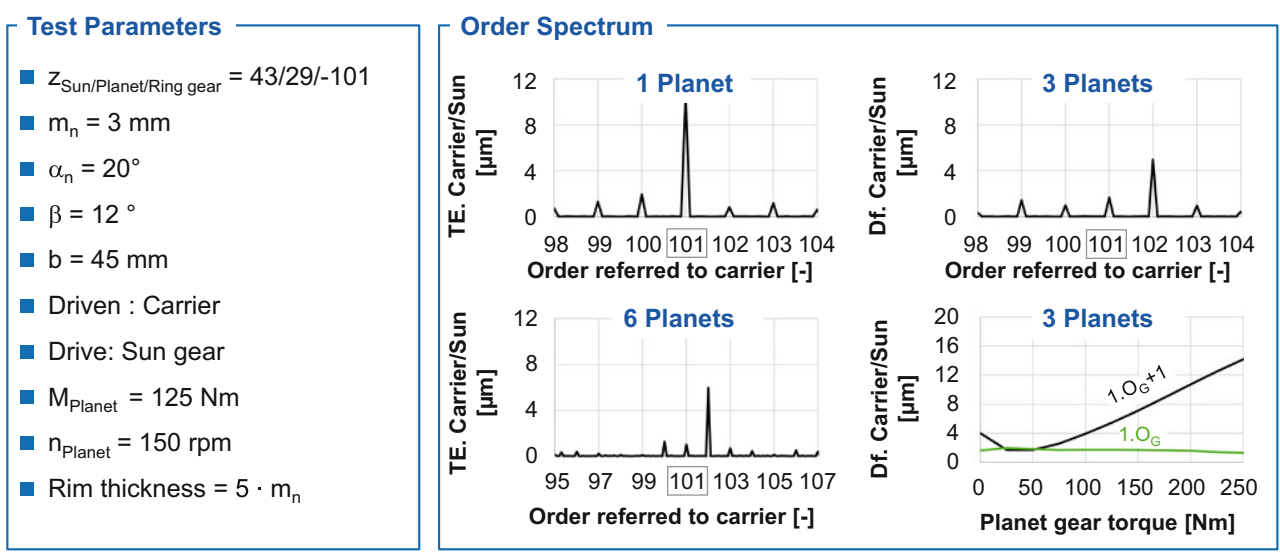

Fig. 7 shows the order spectra of the three different planet gear configurations at a torque of $\mathrm{M}_{\text {Planet }}=125 \mathrm{Nm}$. Due to the number of teeth of the stationary ring gear of $\mathrm{z}_{\mathrm{ring}}=-101$, the first gear mesh order with respect to the planet carrier is $1 . \mathrm{Og}=101$. For the configuration with one planet, a clear excitation of this order can be seen. For three and six planets, the 101st order is hardly excited, but the 102nd order is. This effect occurs over the entire torque range. Comparing the curves of the 101st and 102nd order over the torque for three planets, it can be seen that the 102nd order shows a clear dependence on the torque, while the actual $1 . \mathrm{Oz}$ right.

These observations initially coincide with the results of the structure-borne noise analyses of Inalpolat [1]. He measured for planetary gears with a sequential mesh sequence and evenly spaced planets mainly excitations in orders dividable by the number of planets and not in the 1.Og. The remains low independent of the torque, cf. Fig. 7 bottom
Fig. 8 Comparison of excited and expected orders

Fig. 9 Reasons for modulations in planetary gear stages

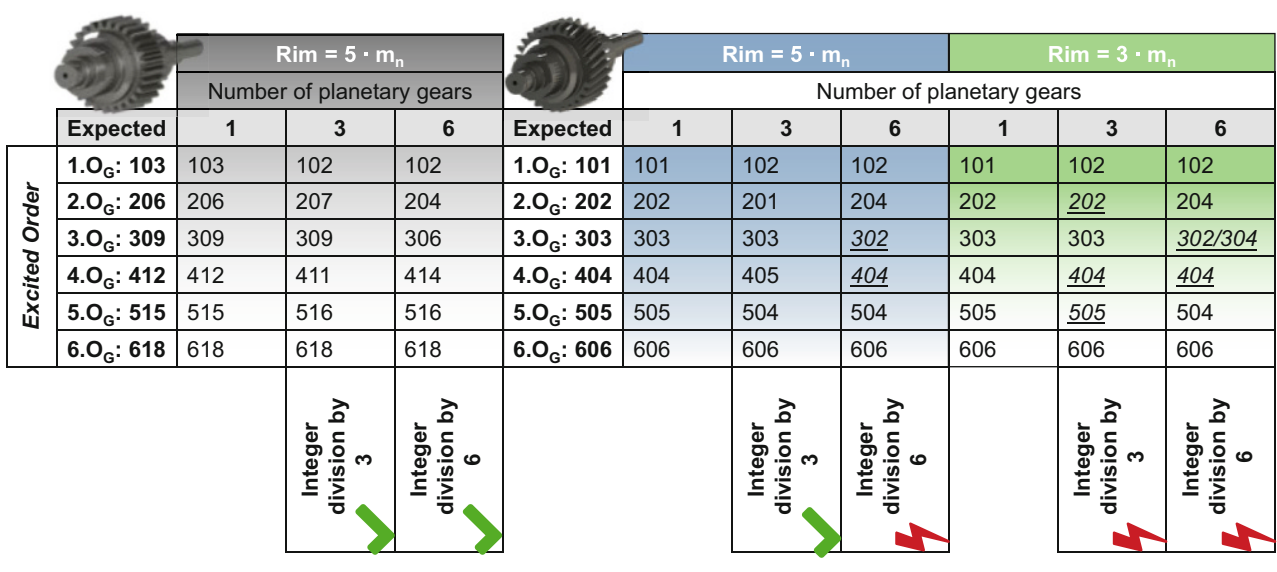
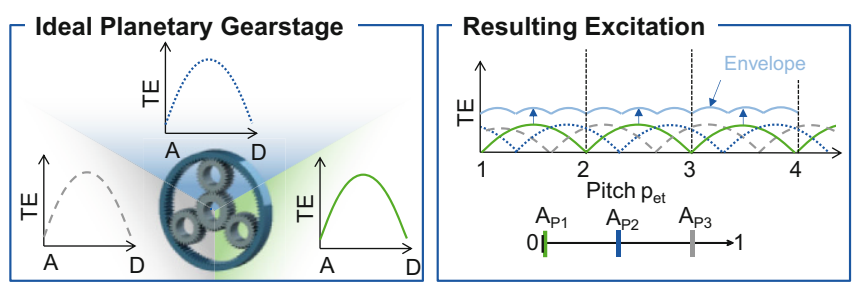

Excited Orders

- At an ideal planetary gearstage with a sequential meshing sequence, the $\mathrm{n}$-th harmonic of the gear mesh order is excited (with $\mathrm{n}=$ Number of planets)

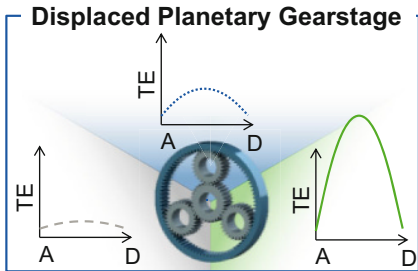

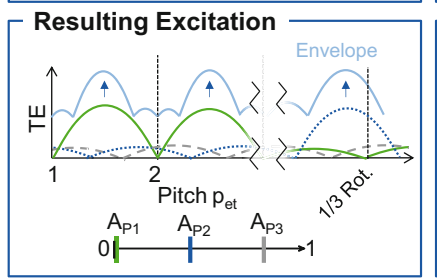

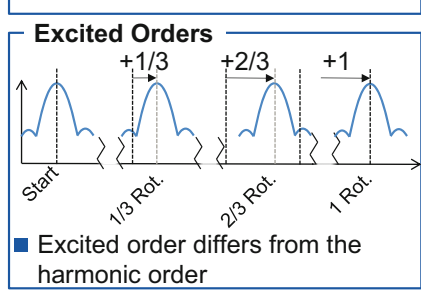


102nd order is in comparison to the 101st order integer dividable by three and six. The observation of the excited orders in the range up to the 6th $\mathrm{Og}$ of all tested reference variants confirms this assumption at least partially, cf. Fig. 8. In the case of the spur gear, all excited orders fulfill the condition according to Inalpolat [1]. The most excited orders deviate from the expected gear mesh order and are integer dividable by the number of planet gears. In the case of the helical variant with a thick-walled ring gear, deviations from this rule can be found for six planets in the $3 . \mathrm{Oz}$ and $4 . \mathrm{Oz}$ range. Even more significant deviations occur with the thin-walled helical ring gear. Here, the nominal gear mesh orders are often excited.

\subsection{Origin of modulations in planetary gearboxes}

The measurement setup of the measuring cell presented here differs significantly from the setup on which Inapolat carried out his investigations [1]. He examines the structureborne noise excitation at a static point of the ring gear and explains the occurring modulations with an amplified excitation by the planet which is closest to the sensor [1].

However, the effect occurring here is measured in the overall transmission error. A local closeness of the planet to the sensor is therefore not admissible as an explanation for the occurring amplitude modulation, since the distance remains constant and is identical for all planets.

When considering an ideal planetary gear stage with a sequential mesh sequence, the transmission errors of all power paths are identical in amplitude, because all sun gear and ring gear meshes react the same, cf. Fig. 9 top left. It is assumed that the sun and ring gear mesh of the planet are in phase. Due to the sequential mesh sequence at the central gears, the individual excitations have a phase shift. The envelope of the individual transmission errors describes the overall transmission error, in which the number of maxima per pitch corresponds to the number of planets. The occurring maxima are all identically high, so that in the case of an ideal sequential planetary gearbox without phase shift at the planet, the nth harmonic of the gear mesh order has the highest excitation, where $n$ corresponds to the number of planet gears.

In a deflected planetary gear stage, the individual amplitudes are not necessarily identical. If, for example, there is an eccentricity of the ring gear, one planet will transmit a higher load than the other two. Due to the higher load, the deformation in this mesh also increases and thus the transmission error. The resulting transmission error then still shows an order component which depends on the number of planets, but there is one dominant excitation per pitch. Due to this effect, the highest excitation shifts into the range of the 1.Og. If the existing deviation is an axis position deviation of the central elements, the planet with the strongest excitation changes depending on the carrier position. Since the individual planets are phase-shifted by one-third pitch due to the sequential meshing in this example, the maximum of the transmission error curve is also phase-shifted by exactly one-third pitch after one-third revolution, see Fig. 9 bottom right. This effect is repeated after a further one-third revolution. If the distance between the individual maxima is increased by one third of a pitch in each case, there is exactly one maximum less than there are meshes after one revolution of the carrier. If the distance between the maxima decrease, the number of maxima would be one more than the number of gear meshes.

The transition between the maximum excitation by one planet to the excitation by the other planet usually takes place fluently, so that it is not possible to clearly assign in the signal whether the observed maximum of the original amplitude is leading by one third pitch or lagging by two third pitches. If the signal is subjected to an FFT, two orders are identified. In this example, the $1 . \mathrm{Og}-1$ and the $1 . \mathrm{Og}+2$, the second with a lower amplitude in comparison. The described effect leads to the fact that the number of maxima and thus also the excited order determined by the FFT always correspond to a multiple of the planet gear number. The excited orders can be calculated with the help of the phase shift at the central gears, Eq. 1.

\section{Equation 1: Calculation of excited orders}

$$
\begin{aligned}
& 1 . \mathrm{Og}+x=\left|z_{\text {central,fixed }}\right|+\left(1-\bmod \left(\frac{z_{\text {central,fixed }}}{n}, 1\right) / n\right) \cdot n \\
& 1 . \mathrm{Og}-y=\left|z_{\text {central,fixed }}\right|-\bmod \left(\frac{z_{\text {central,fixed }}}{n}, 1\right)
\end{aligned}
$$

1.Og First gear mesh order

$x \quad$ Order shift to higher orders

$y \quad$ Order shift to lower orders

$z_{\text {central,fixed }}$ Number of teeth of the fixed central gear

$n \quad$ Number of planets

Modulation effects which could be determined by Inalpolat and Plöger in the structure-borne noise signals at the static ring gear thus also occur similarly in the transmission error $[1,3]$. The cause of the observed order shift is here a combination of an amplitude modulation over one carrier revolution as well as a frequency modulation due to the phase shift. On the test rig, this effect can be explained by a displacement of the ring gear housing due to load-induced deformations of the base plate. This results in a misalignment and center distance deviation between the ring gear and planet carrier axes, which can be measured via the installed displacement sensors. 
Fig. 10 Influence of the planet gear number and the wheel rim thickness
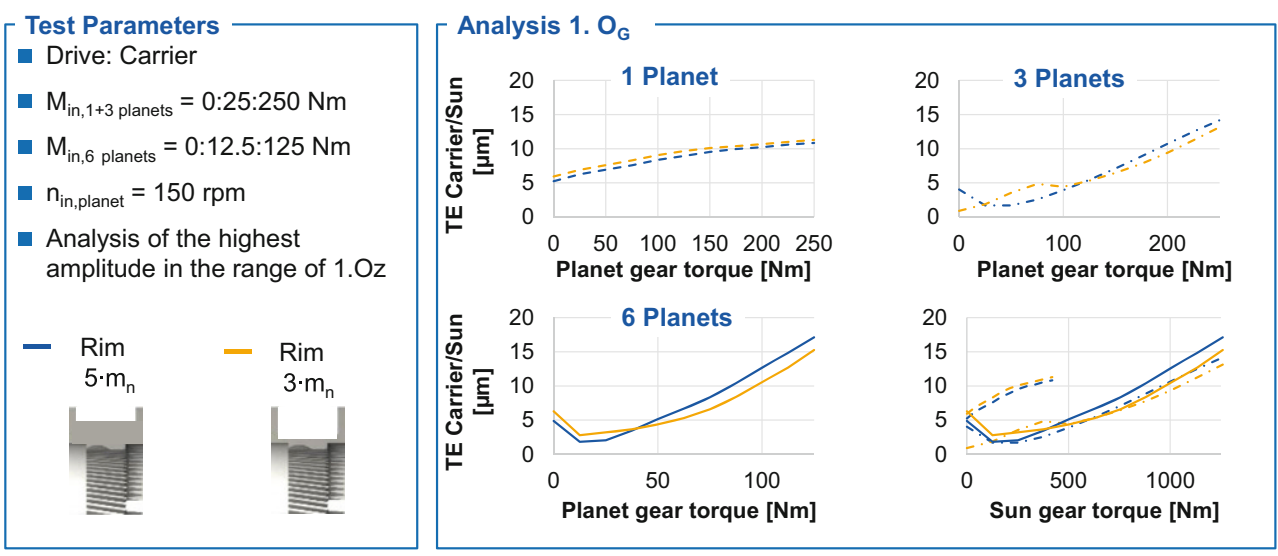

In order for the described effect to occur, certain conditions must be fulfilled:

- An amplitude modulation of the gear mesh order must occur over one carrier revolution.

- The power path causing the highest excitation must vary

- Frequency modulation due to phase-shifted meshing of the central gears must be present.

The measured excitations on the test rig show that the effects also depend on the geometry, cf. Fig. 8. In the case of spur gears, the excitation of the corresponding modulated order also seems to continue at the higher harmonics. This is not the case for helical gears, and in particular for the variant with a thin wheel rim. The positive influence of the helical gear can result from the higher overlap ratio, so that the amplitude increase due to the misalignment is lower. In addition, a thinner gear rim can lead to better load distribution and load sharing in misaligned conditions because of a lower mesh stiffness. Especially a better load sharing between power paths reduces the difference between the excitations of individual power paths, so that the amplitude modulation is further reduced.

\subsection{Reference results without additional deviations}

For the more detailed analysis, the results of the more practically relevant helical gears are mainly used. Another reason is that two different rim thicknesses were manufactured for the helical gear set. The influence of the rim on the excitation in the area of the first gear mesh order for different numbers of planetary gears is shown in Fig. 10. 101st order ( 1 planet) and 102nd order ( 3 and 6 planets) are evaluated. For the two variants with three and six planets, the thinner-walled variant exhibits a slightly lower excitation for higher torques. The comparison of the curves of the individual planet gear configurations shows that for three and six planets, local minima occur in the low load range and the curves increase linearly thereafter. For one planet a degressively increasing curve without minimum can be observed. The different course of one planet in comparison to three and six planets results from the displacement of the sun gear. In the variant with one planet, the sun gear is pushed out of the mesh by the radial force of the planet gear mesh. With three and six planets, the sun is mostly centered by the radial forces of the other planet meshes.

In the lower right diagram, the individual curves are plotted against the sun torque, i.e. the total drive torque, $\mathrm{cf}$. Fig. 10. It can be seen that the curves for three and six planets are very close to each other. This means that the excitation in the area of the first gear mesh order depends in particular on the total torque and not on the individual load of the meshes. This can be caused by deformations of the gearbox that depend on the total torque. So far, it has not been possible to clearly assign this effect to the measured misalignments. The misalignment of the planet carrier due to the load-induced deformation of the base plate shows a clear dependence on the total torque. Another possibility is the torsion of the planet carrier and the associated misalignment of the planet axes. Since the planet carrier torsion cannot be determined by measurement on the test rig, a simulative investigation of the effect is necessary in further work.

\section{Investigation of the influence of additional deviations on the excitation behavior}

Due to the already high misalignments of the sun gear of up to $200 \mu \mathrm{m}$ and the lower industrial relevance of the configuration with only one planet, the analysis of the influence of additional deviations is presented only for the remaining two planet configurations. Shown in Fig. 11 is the 102nd order related to the planet carrier via the related planet gear torque. The reference does not contain any imposed deviations and corresponds to the results from Fig. 10. For better 
Fig. 11 Influence of additional misalignments on the excitation
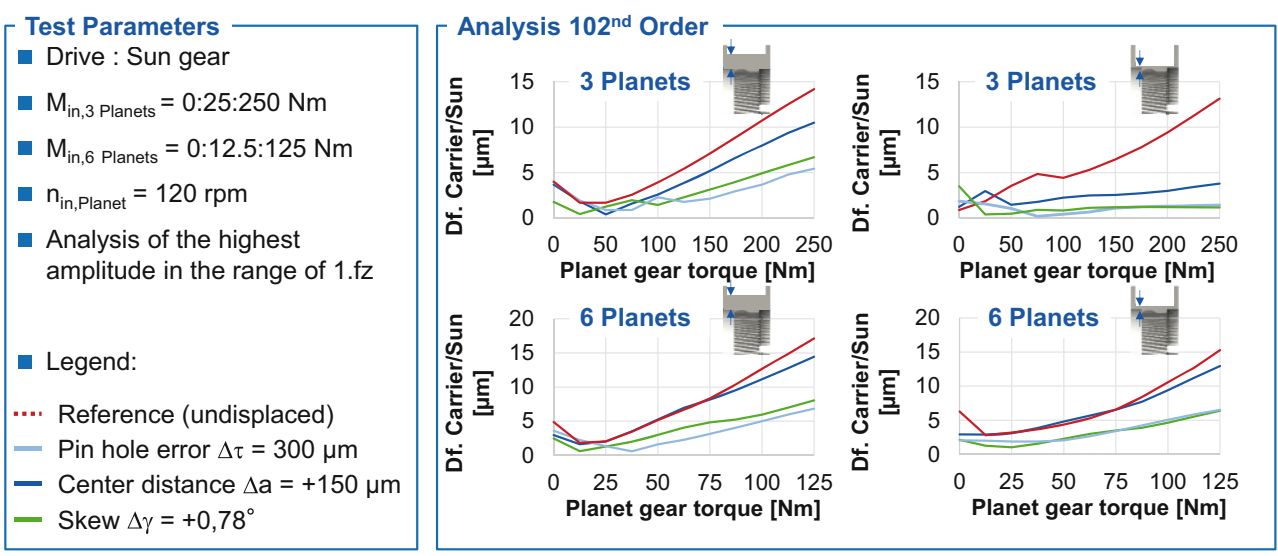

clarity, the results of a pin hole deviation, a larger center distance and a skewed planet gear are shown as examples.

It is noticeable that the reference shows the highest excitations in the range of $1 . \mathrm{Og}$ for all shown variants over. The increased center distance has the next highest excitation and behaves identically to the reference in wide areas. Pin hole error and skew also show similar curves and the lowest excitations for all variants.

The thin-walled ring gear has a positive influence on the excitation in the misaligned condition for three planets. The reason for the better compensation probably lies in the lower mesh stiffness, which makes it easier to compensate misalignments by deforming the mesh. In the case of six planets, the positive effect of the thinner-walled ring gear on the excitation in the 1.Og region is only observed in the case of skewing. This case of misalignment leads to edge contact and a poor load distribution over the tooth width of this mesh. The softer mesh in the thinner-walled variant can better compensate for this effect.

A change in the center distance of a planet gear has the opposite effect on the sun gear and ring gear mesh. The tooth of the planet moves into the gap of the ring gear when the center distance is increased, while it is pulled out of the sun gear mesh. This results in theoretical contact distance changes that lead to penetration in the ring gear mesh and to gaping in the sun gear mesh. This effect is compensated by a rotation of the planet gear within the gear backlash. Since the mesh in the ring gear and the mesh with the sun have the same operating pressure angle, this change in contact distance theoretically balances itself out exactly. However, there are additional changes in the overlap of the respective meshings as well as a small pin hole deviation due to the rotation of the eccentric bushing, which explains the differences to the reference.

For further analysis of the influence of the misalignments, the order spectra are evaluated in the region of the first gear mesh order for the highest torque. The amplitudes of the individual orders for the thick-walled and thin-walled variants for three and six planets are shown, cf. Fig. 12. It can be seen that, in addition to the highest excitation at the 102nd order, further orders are excited at all misaligned conditions compared with the reference, cf. Fig. 7. However, the amplitudes of the resulting sidebands are so low compared with the reduction of the 102nd order that there is no pure distribution of the excitation to further orders, but the excitation itself is reduced, cf. Fig. 12.
Fig. 12 Influence of additional misalignments on occurring side bands

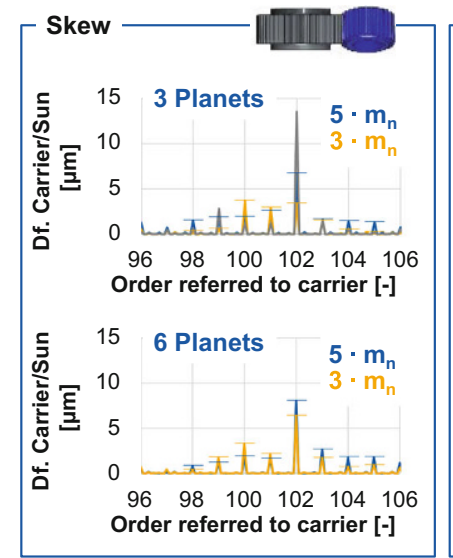

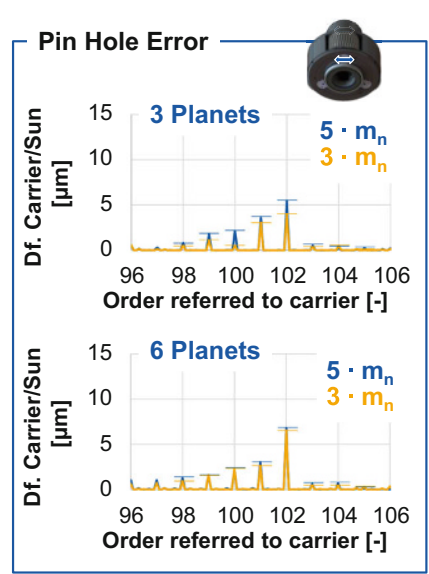


The reduction of the first order and altogether the excitation of the system by applied displacements can only be explained by a misalignment of the system in the reference state which cannot be compensated by a deflection of the sun gear. By additionally misaligning one planet, especially in the configuration with three planets, the space for the sun to relocate gets wider and the gearbox is able to adjust itself. With six planets, this effect is less pronounced, since the remaining five planets continue to keep the sun at a stable position.

The clear positive influence of the thin-walled ring gear at three planets can also be confirmed for the additionally excited orders, so that the excitation is actually reduced and not distributed to other orders. For six planets, there is hardly any difference between the thin-walled and thickwalled ring gear in the analysis of the other orders as well, except for the skewing.

The characteristics of the excited orders differ between the individual misalignments. In the case of the skewing, an increased excitation of the 100th order can additionally be identified as well as lower excitations of the integer orders above and below the 102nd order. When the center distance is increased, this is also observed, but with a much more pronounced excitation of the 102 nd order. For the pin hole deviation, the amplitudes increase continuously from the 98th order up to the 102nd order. For orders above the 102nd order, however, no excitation can be identified anymore.

In addition, it can be stated that in the analysis of the excitation behavior of planetary gearboxes, the exclusive observation of the gear mesh orders is not target-oriented. In the reference state as well as in the misaligned states, almost no excitation would be detected when observing the nominal gear mesh orders due to the order shift to the $102 \mathrm{nd}$ order.

\section{Summary and outlook}

Due to increasingly higher power densities in gear technology, the deformations of the individual components and thus also the misalignments of the tooth contacts increase. Due to the additional influence of the load distribution on the individual power paths, planetary gears react more sensitive to displacements than simple cylindrical gears. In the structure-borne noise signal it was observed, that the measured excitation orders can also deviate from the gear mesh orders due to amplitude and frequency modulations.

A measuring cell is being developed to investigate the influence of elastic deformations and displacements. The analysis of the excitation behavior is carried out by measuring the transmission error at the sun gear, planet gear and planet carrier. Parallel measurements of the displacements of individual elements provide information about their positioning.

The measurement results already show a shift in the excited order of the reference variant. While in theory the third or sixth gear mesh order would have to be excited depending on the number of planet gears, the strongest excitation is determined in the area of the first gear mesh order. A more detailed analysis shows that it is not the actual mesh order that is excited, but an adjacent order. The observation by Inalpolat, that for sequential mesh sequences in structureborne noise especially orders are excited, which are dividable by the number of planets, can also be confirmed for the transmission error [1]. The underlying amplitude modulation results from a displacement of the planet carrier and leads in combination with the frequency modulation by the sequential mesh sequence to a shift of the excited orders.

Thin-walled ring gears have a positive effect on planetary gear boxes with three planets and additional displacements in the tested variants. The measurement results of the additionally displaced variants show a lower maximum excitation in the area of the first gear mesh order and additional sidebands compared to the reference. As a cause for the lower amplitude, a wider space of the sun gear to relocate and a distribution of the excitation energy into the sidebands is assumed.

The modulation effects and the sidebands excited show, that the measurement of individual orders or the exclusive observation of the maximum amplitude in a certain order range is not appropriate. The high displacements shown here, for example, could not be identified in the transmission error signal during an end-of-line test. The results also show that specific displacements could cause a certain characteristic of the order spectrum. In the future, it has to be analyzed whether an identification of misalignment is possible via the excited orders. In addition, a way must be found to rate the excitation of the system when a large number of sidebands are excited by the gear mesh.

Funding Open Access funding enabled and organized by Projekt DEAL.

Open Access This article is licensed under a Creative Commons Attribution 4.0 International License, which permits use, sharing, adaptation, distribution and reproduction in any medium or format, as long as you give appropriate credit to the original author(s) and the source, provide a link to the Creative Commons licence, and indicate if changes were made. The images or other third party material in this article are included in the article's Creative Commons licence, unless indicated otherwise in a credit line to the material. If material is not included in the article's Creative Commons licence and your intended use is not permitted by statutory regulation or exceeds the permitted use, you will need to obtain permission directly from the copyright holder. To view a copy of this licence, visit http://creativecommons.org/licenses/by/4. $0 /$. 


\section{References}

1. Inalpolat M (2009) A theoretical and experimental investigation of modulation sidebands on planetary gear sets. Diss., Ohio State University

2. Morikawa K, Kumagai K, Nishihara R (2014) A study on the sideband phenomenon of planetary gears. Trans JSME. https://doi.org/ 10.1299/transjsme.2014dsm0212

3. Plöger D (2019) Modulation der Zahneingriffsschwingungen von Planetengetrieben. Diss., Technische Universität Darmstadt
4. Theling J, Löpenhaus C, Brecher C (2019) Influence of planet carrier misalignments on the operational behavior of planetary gearboxes. In: Bergs T, Brecher C (eds) 8th WZL Gear Conference in the USA. Apprimus, Aachen, 14-1 bis 14-22

5. DIN ISO (2013) Zylinderräder ISO-Toleranzsystem Teil 1: Definitionen und zulässige Werte für Abweichungen an Zahnflanken (DIN ISO 1328 Part 1)

6. Papies J (2014) Methodik zur systematischen Analyse und Optimierung dynamischer Kraft- und Weganregungen in Planetengetrieben. Diss., Ruhr-Universität Bochum 